\title{
Effects of alcoholism severity and smoking on executive neurocognitive function
}

\author{
Jennifer M. Glass', Anne Buu', Kenneth M. Adams', Joel T. Nigg', Leon I. Puttler², \\ Jennifer M. Jester' \& Robert A. Zucker' \\ University of Michigan, Department of Psychiatry, Substance Abuse Section, Ann Arbor, MI' and Michigan State University, Department of Psychology, East Lansing, \\ MI, USA ${ }^{2}$
}

\section{ABSTRACT}

Aims Neurocognitive deficits in chronic alcoholic men are well documented. Impairments include memory, visualspatial processing, problem solving and executive function. The cause of impairment could include direct effects of alcohol toxicity, pre-existing cognitive deficits that predispose towards substance abuse, comorbid psychiatric disorders and abuse of substances other than alcohol. Cigarette smoking occurs at higher rates in alcoholism and has been linked to poor cognitive performance, yet the effects of smoking on cognitive function in alcoholism are often ignored. We examined whether chronic alcoholism and chronic smoking have effects on executive function. Methods Alcoholism and smoking were examined in a community-recruited sample of alcoholic and non-alcoholic men $(n=240)$ using standard neuropsychological and reaction-time measures of executive function. Alcoholism was measured as the average level of alcoholism diagnoses across the study duration (12 years). Smoking was measured in pack-years. Results Both alcoholism and smoking were correlated negatively with a composite executive function score. For component measures, alcoholism was correlated negatively with a broad range of measures, whereas smoking was correlated negatively with measures that emphasize response speed. In regression analyses, both smoking and alcoholism were significant predictors of executive function composite. However, when IQ is included in the regression analyses, alcoholism severity is no longer significant. Conclusions Both smoking and alcoholism were related to executive function. However, the effect of alcoholism was not independent of IQ, suggesting a generalized effect, perhaps affecting a wide range of cognitive abilities of which executive function is a component. On the other hand, the effect of smoking on measures relying on response speed were independent of IQ, suggesting a more specific processing speed deficit associated with chronic smoking.

Keywords Alcoholism, cigarette smoking, cognition, executive function, response inhibition.

Correspondence to: Jennifer M. Glass, University of Michigan, Department of Psychiatry, Addiction Research Center, 4250 Plymouth Road, Ann Arbor, MI 48109-2700, USA. E-mail: email: jglass@umich.edu

Submitted 5 June 2007; initial review completed 24 October 2007; final version accepted 30 September 2008

\section{INTRODUCTION}

Neurocognitive deficits in men with chronic alcohol use disorders (AUD) are well documented and include visualspatial processing, problem solving, memory and cognitive proficiency [1]. Early theories focused upon frontal lobe dysfunction; recent examinations have focused upon executive function (EF) [2,3], in part because neuroimaging studies have linked AUD to changes in frontal lobe structure and function and their connections to other brain areas [4-6].

Although AUD is known to have neuropsychological consequences linked with brain impairment, the exact mechanism is unclear [7]. Cognitive abilities are related theoretically to substance use onset and outcome, especially EF, because it is linked to poor planning and impulsive behavior $[8,9]$. Thus, some cognitive impairment observed in AUD may have been present before the onset of alcohol use. AUD is also often accompanied by comorbid conditions such as depression or other drug abuse that impact cognition negatively [10].

For example, cigarette smoking occurs at a much higher rate in AUD than in the general population. Surprisingly, the negative effects of smoking on cognition have been ignored until recently [11-13]. Smoking has two potentially different effects: an acute effect of nicotine 
that may be beneficial $[14,15]$ and a chronic negative effect due to long-term smoking. Impairments among smokers compared to non-smokers include general cognitive function [16], working memory [14,17], psychomotor speed [18,19], cognitive flexibility [19] and verbal memory and visual search [20,21]. Glass et al. reported that both smoking and AUD were related negatively to IQ, but smoking mediated the effect of AUD on cognitive proficiency (rapid and accurate cognitive function) [11]. Here the effects of AUD and smoking on EF are examined in a community sample of men with AUD.

$\mathrm{EF}$ is defined as the ability to maintain an appropriate mental set in order to fulfill a future goal [22]. It involves planning, filtering competing information, maintaining a goal despite distraction and inhibiting goal-inconsistent responses. The constructs of intelligence and $\mathrm{EF}$ both involve higher-order cognitive functions and the ability to adapt [23,24]. Measures of EF correlate with IQ, although the correlations are not large [25]. In some models, EF is a component of general intelligence [26]. None the less, intelligence and EF are separable [27], although the extent of this separation remains a point of contention in the literature [26]. In this paper we examine whether chronic AUD and chronic smoking have effects on EF and on response speed that are separable from their effects on IQ. To the extent that EF impairments exist in our sample, they may be specific to EF or part of a more generalized impairment. Furthermore, IQ and EF may correlate more highly in AUD. For example, in comparison to controls, those with AUD used higherorder executive functions to perform a perceptuallearning task [28]. Neuroimaging studies of AUD suggest increased reliance on frontal lobe systems during cognitive tasks $[4,6]$. The recruitment of higher-order functions may be a compensatory strategy that leads to a stronger relationship between EF measures and IQ.

Additionally, most studies of EF in AUD have focused upon neuropsychological tests where performance is based on several component processes. Other tests offer specific focus upon individual components; for example, the Stopping Task uses a reaction-time procedure designed to isolate the ability to inhibit an ongoing behavioral response, an important component of EF [29,30]. The Stopping Task is sensitive to differences in inhibition in many disorders such as attention deficit hyperactivity disorder (ADHD) [31] and cocaine abuse [32], and normal variations in impulsivity [33].

To our knowledge, the Stopping Task has not been tested in AUD, but there is reason to suspect impairment. Children of alcoholic fathers had poorer Stopping Task reaction-times [9], and Stopping Task inhibition predicts the onset of adolescents' alcohol and drug use [8]; a preexisting weakness in response inhibition may be a risk factor for AUD. Furthermore, acute alcohol intake selec- tively disrupts inhibition, but not reaction-time to regular (no stop signal) trials [34]. AUD may lead to long-term impairment in this ability; thus, poor response inhibition may be both a risk for and a consequence of AUD. Because chronic cigarette smoking affects psychomotor and information processing speed $[11,18,19]$, it is expected that smoking will also be associated with slower response inhibition.

In this paper, EF is examined in a communityrecruited sample of men with and without AUD. Executive function impairments in samples from treatment sites have been reported; the current results generalize those findings to a larger population using both traditional neuropsychological measures and reaction-time measures. The effects of smoking on EF in AUD are novel.

\section{METHODS}

\section{Participants}

Data were from 240 men who completed an executive functioning battery as part of wave 5 data collection in the Michigan Longitudinal Study (MLS) [35], a family study of the development of substance use disorders. Families were recruited via men identified through the courts. Men with driving convictions involving a blood alcohol concentration of at least $0.15 \%$ [first conviction, $0.12 \%$ if prior conviction(s)] were potential enrollees if they met diagnosis for probable or definite AUD [36] and had at least one biological son between 3 and 5 years of age (for family risk studies). Non-alcoholic families were recruited by door-to-door canvass in the same neighborhoods as the alcoholic families. This also resulted in recruitment of intermediate-risk families, as some were identified with alcoholic fathers without a history of alcohol-related legal problems. Original recruitment used Feighner criteria [36]; thereafter parents were re-diagnosed using DSM-IV AUD [37] criteria. A detailed description of the study is provided in Zucker et al.[35]. Although some participants sought out-patient treatment, the recruitment pathway for this study was not clinic- or in-patient-based and only $10 \%$ had ever been in treatment. The sample was restricted to men. Although some alcoholic women were also recruited, there are fewer alcoholic women in the sample and the severity of alcoholism is significantly lower, making direct comparisons problematic.

\section{Procedure}

Data were collected in individual homes by trained project staff, blind to diagnostic status, as part of the regular data collection for the MLS. The assessment took 2 hours to complete. Home administration ensured privacy and freedom from distractions. A short questionnaire assessed if there were barriers to collecting valid 
data (e.g. lack of sleep, immediately prior use of alcohol, recreational or prescription drugs). If two or more alcoholic drinks were taken within the hour prior to testing, or the person appeared intoxicated or 'high', the assessment was rescheduled. Other than self-report and the judgement of the examiner; no other drug screens were employed. Participants who smoked were allowed to smoke cigarettes prior to testing and during breaks. Breaks were taken on an as-requested basis at the end of each test, thus most participants were not in a state of nicotine withdrawal; however, a formal test of nicotine withdrawal was not included.

The specific tests were selected to address our hypotheses with a combination of instruments having strong psychometric properties. The focus was EF, construed broadly as a multi-faceted construct [9,22].

\section{Measures of executive control}

\section{Stroop Color and Word Test (Stroop Test)}

The Stroop Test [38] is a timed task in which the participant has to read through three sets of stimuli (words and colors). For word-reading, color names are printed in black ink, for color-naming a string of Xs (XXXX) are printed in colored ink. For the interference set, color names are printed in colored ink; the participant must name the color of the ink and resist interference from the printed color word (e.g. the word blue printed in red ink). Reported are the number of items completed correctly in a 90-second interval per set.

\section{Controlled Oral Word Association Test (COWAT)}

The COWAT [38] is a timed test of verbal word fluency. Participants are asked to generate as many words as they can that begin with various letters of the alphabet during a 1-minute period. The score that the participant receives is the sum of all acceptable words produced during three trials.

\section{Wisconsin Card Sorting Test (WCST [38])}

The WCST consists of four stimulus cards and 64 response cards that depict figures of varying forms, colors and numbers of figures. Participants are told to match each consecutive card from the deck with one of the four stimulus cards. The participant is told whether his/her response is correct or wrong, but is not told the sorting principle involved. The participant must match the sorting principle (color, form or number) for a specified number of responses before the sorting principle is changed. For this study we used the computer version of the instrument. Reported are the number of categories achieved and number of perseverative errors.
Paced Auditory Serial Addition Test (PASAT [38])

The PASAT measures verbal working memory, attention, concentration and speed of information processing. The test requires the participant to add randomized digits so that each is added to the digit immediately preceding it. The digits are presented at four rates of speed. A taped representation is used to provide precise control over the rate at which stimuli are presented. Reported are the total number of correct responses.

\section{Trail Making Test (TMT [38])}

The TMT is a widely used measure of visual conceptual and visual-motor tracking as well as set-switching. TMT consists of two parts, with sequential circles containing ascending numbers (part A) and alternating numbers and letters (part B). Participants draw lines between the sequential circles, with the examiner pointing out errors to the participant as they occur. If an error occurs, the participant corrects the error and continues the task. Reported are the times taken to complete each part.

\section{Stopping task [29,30]}

During this two-alternative choice reaction-time task, participants see an $\mathrm{X}$ or an $\mathrm{O}$ on a computer screen and respond rapidly with one of two keys. On some trials a tone sounds shortly after the X or $\mathrm{O}$ appears, indicating that participants should withhold a response. After two practice blocks of 32 trials each, four blocks of 64 trials are administered. The final three blocks are averaged unless data quality checks suggest otherwise [31]. The most reliable estimates of stop signal reaction-time (SSRT) are obtained with a response-reaction-time tracking methodology [39] where the delay between the visual stimulus and the warning tone is varied to maintain 50\% success rate at withholding the response. A quantitative model of reaction-time (RT) processes enables calculation of each participant's speed of stopping or inhibiting a response (SS-RT) by subtracting average stop signal delay from average RT to the trials without a stop signal (GoRT) [29]. Go-RT trials with incorrect responses were not included in the calculation of Go-RT. This SS-RT estimate is a measure of inhibitory control. The Go-RT and variability of Go-RT are measures of response activation.

\section{Measures of substance abuse}

\section{Alcoholism severity}

Due to the nature of our sample, the assignment of participants to either control or alcoholic group was not always clear. For example, some participants met diagnostic criteria for AUD early in life, but not at the time of testing. To address diagnostic variation over time, we 
developed a continuous variable of alcohol severity. The alcoholism severity index was computed by averaging across T1 (baseline) to T5 (12 years). The severity at each wave was coded as: 0 for negative diagnosis; 1 for alcohol abuse; 2 for alcohol dependence without physical dependence; and 3 for alcohol dependence with physical dependence. The resulting index is a continuous scale ranging from 0 to 3 .

\section{Monthly drinking rate}

A monthly drinking rate for each participant (see Table 1) was calculated based on the Drinking and Drug History Questionnaire number of days each month where alcohol is consumed multiplied by the average number of drinks consumed per day on a day when alcohol is consumed over the past 6 months).

\section{Cigarette smoking}

Rate of smoking was obtained from the Drinking and Drug History Questionnaire. At the time of testing, $41.5 \%$ reported regular smoking. A continuous variable, called 'pack-years', was created by multiplying average daily use (in packs) by the number of years smoking (see Table 1). Daily use was estimated from self-reported rate of smoking from study waves 1-5. Years of smoking was estimated from self-reported age at smoking onset to current age. Among those who had ever smoked, average pack-years were 20.17 years.

\section{Other drug use}

Use of drugs other than alcohol or cigarettes was obtained from the Drinking and Drug History Questionnaire. Participants were asked on how many occasions during the last 3 years they had used: marijuana, lysergic acid diethylamide (LSD), psychedelics other than LSD, cocaine, amphetamines, Quaaludes, barbiturates, tranquilizers, heroin, narcotics other than heroin or sniffed glue. Marijuana had the highest frequency of use, with $22.2 \%$ of the sample reporting any use, followed by cocaine $(6.1 \%)$ and tranquilizers $(5.9 \%)$. A composite variable of any drug use was computed by summing the frequency of use for each of the individual drug categories for each participant (see Table 1). For the total druguse score, $24.5 \%$ of the sample reported some use in the past 3 years.

\section{Psychiatric symptoms}

Depression

Depression at the time of testing was measured by clinician ratings using the Hamilton Depression Scale [40].

\section{Antisocial personality disorder (ASPD)}

ASPD was assessed via interview using the Diagnostic Interview Schedule-version IV (DIS-IV) [41]. Diagnosis was based on wave 1 only because this disorder, by definition, yields a life-time diagnosis. The diagnosis was based on the DIS, supplemented by information provided by the 46-item self-report Antisocial Behavior Inventory [42] which assesses the frequency of aggressive and antisocial activity in childhood and adulthood.

Attention deficit hyperactivity disorder symptom count (ADHDs $x)$

This measure was a simple count of all ADHD symptom questions on the DIS-IV, divided by the maximum possible score of 18 symptoms.

Table 1 Demographics, drinking rate, smoking and other drug by alcoholism severity group.

\begin{tabular}{|c|c|c|c|c|}
\hline & $\begin{array}{l}\text { Group } 1 \\
n=89\end{array}$ & $\begin{array}{l}\text { Group } 2 \\
n=65\end{array}$ & $\begin{array}{l}\text { Group } 3 \\
n=53\end{array}$ & $\begin{array}{l}\text { Group } 4 \\
n=33\end{array}$ \\
\hline Age & $46.2(6.1)$ & $43.8(5.1)$ & $44.3(4.8)$ & $44.8(4.7)$ \\
\hline Education & $14.7(2.3)$ & $14.0(2.8)$ & $13.5(2.3)$ & $13.9(2.3)$ \\
\hline IQ & $110.8(13.0)$ & $105.6(12.0)$ & $100.8(10.4)$ & $104.3(14.5)$ \\
\hline Depression & $4.4(4.9)$ & $6.7(7.0)$ & $6.1(6.2)$ & $6.9(7.4)$ \\
\hline ASPD & $7 \%$ & $12 \%$ & $33 \%$ & $35 \%$ \\
\hline ADHDsx & $0.04(0.14)$ & $0.11(0.25)$ & $0.07(0.17)$ & $0.06(0.15)$ \\
\hline Smoking (PkYrs) & $5.6(17.0)$ & $10.8(15.3)$ & $11.9(15.1)$ & $19.4(17.6)$ \\
\hline Other drug use & $0.4(1.5)$ & $2.1(4.4)$ & $2.3(3.4)$ & $6.7(7.9)$ \\
\hline Drinking rate months) & $5.8(11.6)$ & $32.5(59.3)$ & $63.9(111.5)$ & $104.3(92.6)$ \\
\hline
\end{tabular}

Group 1: severity = 0; group 2: $0<$ severity $\leq 1$; group 3: $1<$ severity $\leq 2$; group 4: $2<$ severity $\leq 3$; ASPD: antisocial personality disorder, percentage with diagnosis; ADHDsx: attention deficit hyperactivity disorder, symptom count percentage; IQ: intelligence quotient; PkYrs: pack-years; other drug use: sum of reported use of drugs other than alcohol or nicotine over the past 3 years; drinking rate: over the past 6 months, the average number of alcoholic drinks normally consumed in a month; data are mean (standard deviation), unless otherwise not. 


\section{Education}

The highest level of education achieved at the time of testing was calculated as years of education.

\section{Intelligence quotient (IQ)}

IQ, one of the most widely accepted and psychometrically well-established indices of intellectual functioning [24], was estimated with a four-subtest short form of the Weschler Adult Intelligence Scale-revised (WAIS-R) [43] consisting of information, picture completion, arithmetic and block design.

\section{Data analyses}

Each variable was standardized (using the sample mean and standard deviation for each individual variable) and if necessary reverse-coded. An EF composite variable was created by averaging each of the variables for each participant.

\section{Missing data}

There were about $10 \%$ missing data across variables. Multiple imputation proposed by Rubin was adopted to deal with missing data [44]. We assumed 'missing at random' (i.e. the probability of response does not depend on missing values) that was satisfied by the protocol of MLS data collection. SAS PROC MI was used to generate five plausible alternative versions of the complete data. Each of the five data sets was analyzed in the same fashion by a complete data method. The five sets of results were then aggregated by using SAS PROC MIANALYZE to obtain overall estimates and standard errors that reflect missing data uncertainty and finite sample variation.

\section{Statistical analysis}

We examined zero-order correlations between smoking, alcoholism severity and EF; first with the composite variable and then, if significant, with the component measures. All probabilities are one-tailed.

To test the relationship between alcoholism severity, smoking and EF, we conducted a series of linear regression analyses using SAS PROC REG on each of the five plausible data sets generated by SAS PROC MI. SAS PROC MIANALYZE was used to aggregate the five sets of results. We started with the executive-function composite; if significant, we followed-up with regression analyses using component measures. For each EF variable, we tested up to four different regression models. Predictors were entered simultaneously for each of the models.

Model 1 investigated whether alcoholism severity and smoking were independent predictors of EF by including both severity and smoking in the regression model. Note that this model was tested only if both smoking and alcoholism severity were correlated significantly with the particular EF measure.

Model 2 added education to model 1, as education was lower in the alcoholic group. Some differences associated with alcoholism could be due simply to lower education attainment rather than dysfunction caused by alcoholism. However, given the life-style associated with alcoholism there may be fewer opportunities for education, and therefore lower education is part of the syndrome. To examine whether alcoholism causes brain dysfunction, education should be included to control for its effects. To examine the overall consequences of alcoholism, controlling for education may underestimate the total effect. Because this is a descriptive paper documenting executive function in a community-based sample, we present our analyses both with and without education included as a predictor.

Model 3 added IQ to model 1. As with education, whether or not to include IQ as a factor can be argued in either direction. It is theoretically interesting to know whether effects of alcoholism or smoking on EF are separable from IQ. Thus, we present our regression analyses both with and without IQ included in the models.

Model 4 added the Hamilton current depression score to model 1, as higher scores on depression were related to lower performance on the EF composite score and to four of the individual measures.

Finally, we tested whether EF and IQ become more correlated with increasing alcoholism severity by creating two separate dummy variables based on severity. One variable coded diagnosis of abuse or higher as 1 (no diagnosis was coded as 0 ). The other variable coded diagnosis of dependence as 1 and abuse or no diagnosis as 0 . These two variables were included in a regression model along with IQ and the interaction between IQ and the dummy variables to predict $\mathrm{EF}$ (composite).

\section{RESULTS}

Means for the demographic, psychiatric and drinking, smoking and drug use measures by the four alcoholism severity groups are presented in Table 1 . To present the data in tabular form, we divided the participants into four groups: alcoholism severity equals zero, less than or equal to one, less than or equal to two, and less than or equal to three.

Diagnosis of ASPD was more common in the highest two alcoholism severity groups. Further analysis showed higher level of smoking (pack-years) among those with an ASPD diagnosis [mean $=21.3$, standard deviation $(\mathrm{SD})=19.0]$ than those without the diagnosis (mean $=$ 8.2, $\mathrm{SD}=15.6)$. This difference was significant, $t_{(222)}=$ 4.618, $P<0.001$. There was also a higher monthly 
Table 2 Correlations of alcoholism severity, drinking rate, smoking and other drugs with executive function measures.

\begin{tabular}{|c|c|c|c|c|}
\hline & Alcoholism severity & Drinking rate & Smoking (PkYrs) & Other drug \\
\hline \multicolumn{5}{|c|}{ Executive function composite } \\
\hline & $0.243^{* * *}$ & -0.015 & $-0.236^{* * *}$ & -0.019 \\
\hline COWAT & $-0.115^{*}$ & 0.020 & 0.038 & 0.185 \\
\hline PASAT & $-0.221^{* * *}$ & 0.003 & -0.080 & -0.051 \\
\hline STROOP-w & $-0.143^{* *}$ & 0.057 & $-0.162^{* *}$ & -0.087 \\
\hline STROOP-c & $-0.154^{* *}$ & 0.032 & $-0.130^{*}$ & 0.006 \\
\hline STROOP-cw & $-0.119^{*}$ & -0.032 & -0.056 & 0.013 \\
\hline WCST-pe & -0.045 & -0.025 & -0.050 & 0.082 \\
\hline WCST-cat & $-0.113^{*}$ & -0.089 & -0.078 & 0.044 \\
\hline TMT-A & $-0.195^{* * *}$ & 0.030 & $-0.229^{* * *}$ & -0.024 \\
\hline TMT-B & -0.095 & 0.006 & $-0.213^{* * *}$ & 0.048 \\
\hline Go-RT & $-0.181^{* *}$ & -0.019 & $-0.262^{* * *}$ & -0.070 \\
\hline Go-RT var. & $-0.194^{* * *}$ & -0.077 & $-0.254^{* * *}$ & -0.059 \\
\hline SS-RT & $-0.167^{* *}$ & -0.018 & $-0.221^{* * *}$ & $-0.119^{*}$ \\
\hline
\end{tabular}

PkYrs: pack-years; COWAT: Controlled Oral Word Association Test; PASAT: Paced Auditory Serial Addition Task; STROOP-w: Stroop Word Reading Test; STROOP-c: Stroop Color Naming Test; STROOP-cw: Stroop Color Word Interference Test; WCST-pe: Wisconsin Card Sorting Test, perseverative errors; WCST-cat: Wisconsin Card Sorting Test, categories achieved; TMT-A: Trail Making Test A; TMT-B: Trail Making Test B; Go-RT: Stopping Task go trials reaction-time; Go-RT var.: Stopping Task go trial reaction-time variability; SS-RT: Stopping Task stopping reaction-time. ${ }^{*} P<0.05 ;{ }^{* *} P<0.01$; ${ }^{* * *} P<0.001$.

drinking rate among those with an ASPD diagnosis (mean $=60.1, \mathrm{SD}=135.3)$ than among those without the diagnosis $($ mean $=31.3, \mathrm{SD}=51.8), t_{(208)}=2.144$, $P<0.034$.

\section{Correlations}

Alcoholism was correlated significantly with smoking $(r=0.255, \quad P<0.001)$, other drug use $(r=0.363$, $P<0.001)$, education $(r=-0.181, \quad P<0.01)$, IQ $(r=-0.211, \quad P<0.01)$ and depression $(r=0.1095$, $P<0.05)$, but not ADHD symptoms $(r=0.034)$. Smoking was correlated significantly with other drug use $(r=0.137, P<0.05)$, education $(r=-0.219, P<0.001)$ and IQ $(r=-0.195, P<0.01)$, but not depression $(r=0.020)$ or ADHD symptoms $(r=0.072)$.

Table 2 shows the zero-order correlations between the EF measures and alcoholism severity, monthly drinking rate, smoking and other drug use. Alcoholism severity and monthly drinking rate were correlated significantly, but only moderately $(r=0.467, P<0.001)$. Alcoholism severity, smoking, education, depression and IQ were all correlated significantly with the EF composite score. For the individual EF scores, alcoholism severity was correlated moderately negatively with at least one measure from each of the tests, but monthly drinking rate was not correlated with any of the EF measures. Therefore, our subsequent analyses focused upon alcoholism severity rather than drinking rate. Smoking, on the other hand, showed consistent correlations with tests that emphasize response speed. Other drug use was not correlated with any EF measure except SS-RT.
Table 3 Correlations of education, depression and IQ with executive function.

\begin{tabular}{llll}
\hline & Education & Depression & IQ \\
\hline Executive function composite & & \\
& $0.353^{* * *}$ & $-0.144^{* *}$ & $0.590^{* * *}$ \\
COWAT & $0.318^{* * *}$ & -0.056 & $0.554^{* * *}$ \\
PASAT & $0.408^{* * *}$ & -0.076 & $0.468^{* * *}$ \\
STROOP-w & $0.282^{* * *}$ & -0.091 & $0.342^{* * *}$ \\
STROOP-c & $0.199^{* * *}$ & -0.066 & $0.321^{* * *}$ \\
STROOP-cw & $0.217^{* * *}$ & $-0.113^{*}$ & $0.395^{* * *}$ \\
WCST-pe & $0.219^{* * *}$ & -0.073 & $0.302^{* * *}$ \\
WCST-cat & $0.165^{* *}$ & -0.037 & $0.203^{* * *}$ \\
TMT-A & $0.120^{*}$ & $-0.187^{* *}$ & $0.374^{* * *}$ \\
TMT-B & $0.244^{* * *}$ & $-0.120^{*}$ & $0.467^{* * *}$ \\
Go-RT & 0.082 & -0.061 & $0.156^{* *}$ \\
Go-RT var. & $0.184^{* *}$ & $-0.108^{*}$ & $0.331^{* * *}$ \\
SS-RT & 0.099 & -0.044 & $0.294^{* * *}$ \\
& & &
\end{tabular}

COWAT: Controlled Oral Word Association Test; PASAT: Paced Auditory Serial Addition Task; STROOP-w: Stroop Word Reading Test; STROOP-c: Stroop Color Naming Test; STROOP-cw: Stroop Color Word Interference Test; WCST-pe: Wisconsin Card Sorting Test, perseverative errors; WCSTcat: Wisconsin Card Sorting Test, categories achieved; TMT-A: Trail Making Test A; TMT-B: Trail Making Test B; Go-RT: Stopping Task go trials reaction-time; Go-RT var:: Stopping Task go trial reaction-time variability; SS-RT: Stopping Task stopping reaction-time; IQ: intelligence quotient. ${ }^{*} P<0.05,{ }^{* *} P<0.01,{ }^{* * *} P<0.001$

Table 3 shows the zero-order correlations between the EF measures and education, depression and IQ, which were all correlated significantly with the EF composite score. Education was correlated positively with all the EF measures except Go-RT and Go-RT variability. Depression was correlated with the Stroop color-word task, TMT-A 
Table 4 Standardized means (standard deviations) for executive function measures by alcoholism severity group.

\begin{tabular}{|c|c|c|c|c|}
\hline & $\begin{array}{l}\text { Group } 1 \\
n=89\end{array}$ & $\begin{array}{l}\text { Group } 2 \\
n=65\end{array}$ & $\begin{array}{l}\text { Group } 3 \\
n=53\end{array}$ & $\begin{array}{l}\text { Group } 4 \\
n=33\end{array}$ \\
\hline Composite & $0.12(0.57)$ & $0.07(0.56)$ & $-0.17(0.60)$ & $-0.19(0.67)$ \\
\hline COWAT & $0.15(0.95)$ & $-0.06(0.96)$ & $-0.14(0.93)$ & $-0.02(1.19)$ \\
\hline PASAT & $0.17(0.97)$ & $0.17(1.10)$ & $-0.40(0.96)$ & $-0.35(0.93)$ \\
\hline STROOP-w & $0.11(1.00)$ & $0.09(1.05)$ & $-0.15(0.93)$ & $-0.17(0.96)$ \\
\hline STROOP-c & $0.12(0.96)$ & $0.06(1.03)$ & $-0.15(0.98)$ & $-0.21(1.11)$ \\
\hline STROOP-cw & $0.06(1.00)$ & $0.08(1.06)$ & $-0.09(0.94)$ & $-0.20(0.89)$ \\
\hline WCST-pe & $-0.06(1.15)$ & $0.15(0.75)$ & $-0.03(0.84)$ & $-0.09(1.20)$ \\
\hline WCST-cat & $0.02(0.97)$ & $0.17(0.81)$ & $-0.12(1.08)$ & $-0.22(1.25)$ \\
\hline TMT-A & $0.19(1.02)$ & $0.18(0.81)$ & $-0.36(1.14)$ & $-0.18(0.99)$ \\
\hline TMT-B & $0.11(1.16)$ & $0.06(0.90)$ & $-0.25(0.81)$ & $-0.03(0.97)$ \\
\hline Go-RT & $0.19(0.90)$ & $-0.04(1.00)$ & $-0.13(1.09)$ & $-0.31(1.00)$ \\
\hline Go-RT var. & $0.20(0.94)$ & $-0.06(1.04)$ & $-0.13(1.09)$ & $-0.30(0.89)$ \\
\hline SS-RT & $0.17(0.94)$ & $0.04(0.92)$ & $-0.15(1.02)$ & $-0.24(1.16)$ \\
\hline
\end{tabular}

Group 1: severity = 0; group 2: $0<$ severity $\leq 1$; group 3: $1<$ severity $\leq 2$; group 4: $2<$ severity $\leq$ 3; COWAT: Controlled Oral Word Association Test; PASAT: Paced Auditory Serial Addition Task; STROOP-w: Stroop Word Reading Test; STROOP-c: Stroop Color Naming Test; STROOP-cw: Stroop Color Word Interference Test; WCST-pe: Wisconsin Card Sorting Test, perseverative errors; WCST-cat: Wisconsin Card Sorting Test, categories achieved; TMT-A: Trail Making Test A; TMT-B: Trail Making Test B; Go-RT: Stopping Task go trials reaction-time; Go-RT var.: Stopping Task go trial reaction-time variability; SS-RT: Stopping Task stopping reaction-time.

and -B and Go-RT variability. IQ was correlated with all the EF measures.

Table 4 shows the standardized EF means for each of the alcohol severity groups. For most of the measures, the means show decreasing performance with increasing alcoholism severity.

\section{Regression models}

Table 5 shows the regression coefficients for the four models described above for each of the EF measures. The alcoholism $\times$ smoking interaction was not significant and was dropped from all models. It should also be noted that models were tested for each measure only if the predictors to be included in the model had a significant zeroorder correlation with that measure.

For model 1, alcoholism severity and smoking remained significant predictors of the $\mathrm{EF}$ composite, Stroop word reading, TMT-A, Go-RT, Go-RT variability and SS-RT, indicating that for these measures alcoholism and smoking have independent effects. For Stroop color naming, smoking dropped out; for this measure alcoholism mediated the effect of smoking.

For model 2 (education), alcoholism severity and smoking remained significant predictors of the EF composite, TMT-A and Go-RT variability. Thus, even with education included, both smoking and alcoholism were significant predictors of general EF and for some measures with an emphasis on response speed. However, the effects of smoking and alcoholism severity were reduced to non-significant levels for Stroop word reading and Stroop color naming. The effects of alcoholism severity were reduced to non-significant levels for COWAT, Stroop color-word and WCST categories. Alcoholism severity remained a significant predictor of PASAT.

For model 3 (IQ), the effects of alcoholism severity drop to non-significant levels for all measures, including the EF composite. On the other hand, smoking (packyears) remained a significant predictor for the composite, TMT-A, Go-RT, Go-RT variability and SS-RT. Thus, even with IQ included, smoking continued to be a significant predictor of performance on tasks with an emphasis on response speed.

For model 4 (depression), alcoholism severity and smoking (pack-years) remained significant predictors for the composite, TMT-A and Go-RT variability.

There was no evidence that EF and IQ became more correlated with increasing severity; the interaction terms between IQ and severity dummy variables were not significant $(P>0.06)$.

Because ASPD and ADHD are linked theoretically and empirically to EF and because ASPD was related significantly to alcoholism severity and drinking rate, as well as smoking, we also conducted separate regression analyses using the $\mathrm{EF}$ composite to be sure that our results were not due to the neurocognitive effects of these psychiatric measures. In a regression model with ASPD diagnosis, alcoholism severity and smoking (pack-years), ASPD was not significant [coeff. $=-0.132$, standard error $(\mathrm{SE})=0.110 ; t=-1.19, P=0.23]$, while both alcoholism severity (coeff. $=-0.118, \mathrm{SE}=0.045 ; t=-2.64$, $P<0.009)$ and smoking (pack-years) (coeff. $=-0.006$, $\mathrm{SE}=0.002 ; t=-2.30, P<0.023)$ remained significant. In a similar model with ADHD symptoms, ADHD was 
Table 5 Regression models predicting executive function.

\begin{tabular}{|c|c|c|c|c|}
\hline & $\begin{array}{l}\text { Model } 1 \\
\text { Coeff. (SE) }\end{array}$ & $\begin{array}{l}\text { Model } 2 \\
\text { Coeff. (SE) }\end{array}$ & $\begin{array}{l}\text { Model } 3 \\
\text { Coeff. (SE) }\end{array}$ & $\begin{array}{l}\text { Model } 4 \\
\text { Coeff. (SE) }\end{array}$ \\
\hline \multicolumn{5}{|l|}{ Composite } \\
\hline Alc. severity & $-0.127^{* * *}(0.042)$ & $-0.095^{* *}(0.040)$ & $-0.047(0.035)$ & $-0.119^{* *}(0.061)$ \\
\hline Smoking (PkYrs) & $-0.007 * *(0.002)$ & $-0.005 *(0.002)$ & $-0.004 *(0.002)$ & $-0.007^{* *}(0.002)$ \\
\hline Education & - & $0.069^{* * *}(0.015)$ & - & - \\
\hline IQ & - & - & $0.027^{* * *}(0.002)$ & \\
\hline Depression & - & - & - & $-0.011^{*}(0.006)$ \\
\hline \multicolumn{5}{|l|}{ COWAT } \\
\hline Alc. severity & - & $-0.081(0.070)$ & $-0.011(0.062)$ & - \\
\hline Education & - & $0.114^{* * *}(0.027)$ & - & - \\
\hline $\mathrm{IQ}$ & - & - & $0.044^{* * *}(0.006)$ & - \\
\hline \multicolumn{5}{|l|}{ PASAT } \\
\hline Alc. severity & - & $-0.155^{*}(0.072)$ & $-0.102(0.068)$ & - \\
\hline Education & - & $0.156^{* * *}(0.027)$ & - & - \\
\hline IQ & - & - & $0.039 * * *(0.006)$ & - \\
\hline \multicolumn{5}{|l|}{ STROOP-w } \\
\hline Alc. severity & $-0.123 *(0.090)$ & $-0.085(0.071)$ & $-0.049(0.072)$ & - \\
\hline Smoking (PkYrs) & $-0.008 *(0.004)$ & $-0.005(0.004)$ & $-0.005(0.004)$ & - \\
\hline Education & - & $0.103^{* * *}(0.026)$ & - & - \\
\hline IQ & - & - & $0.026^{* * *}(0.005)$ & - \\
\hline \multicolumn{5}{|l|}{ STROOP-c } \\
\hline Alc. severity & $-0.137 *(0.072)$ & $-0.113(0.072)$ & $-0.059(0.070)$ & - \\
\hline Smoking (PkYrs) & $-0.006(0.004)$ & $-0.004(0.004)$ & $-0.003(0.002)$ & - \\
\hline Education & - & $0.065^{* *}(0.027)$ & - & - \\
\hline IQ & - & - & $0.028^{* * *}(0.005)$ & - \\
\hline \multicolumn{5}{|l|}{ STROOP-cw } \\
\hline Alc. severity & - & $-0.081(0.070)$ & $-0.049(0.012)$ & $-0.114(0.074)$ \\
\hline Education & - & $0.083^{* * *}(0.027)$ & - & - \\
\hline IQ & - & - & $0.032^{* * *}(0.005)$ & - \\
\hline Depression & - & - & - & $-0.014(0.011)$ \\
\hline \multicolumn{5}{|l|}{ WCST-cat } \\
\hline Alc. severity & - & $-0.077(0.072)$ & $-0.057(0.072)$ & - \\
\hline Education & - & $0.071^{* *}(0.027)$ & - & - \\
\hline IQ & - & - & $0.016^{* * *}(0.005)$ & - \\
\hline \multicolumn{5}{|l|}{ TMT-A } \\
\hline Alc. severity & $-0.153 *(0.074)$ & $-0.121 *(0.074)$ & $-0.056(0.071)$ & $-0.132 *(0.074)$ \\
\hline Smoking (PkYrs) & $-0.011^{* *}(0.004)$ & $-0.010^{* *}(0.004)$ & $-0.008 *(0.004)$ & $-0.011^{* *}(0.010)$ \\
\hline Education & - & $0.030(0.027)$ & - & - \\
\hline $\mathrm{IQ}$ & - & - & $0.029 * * *(0.005)$ & - \\
\hline Depression & & - & - & - \\
\hline \multicolumn{5}{|l|}{ Go-RT } \\
\hline Alc. severity & $-0.140 *(0.075)$ & - & $-0.115(0.079)$ & - \\
\hline Smoking (PkYrs) & $-0.014^{* * *}(0.004)$ & - & $-0.013^{* * *}(0.004)$ & - \\
\hline $\mathrm{IQ}$ & - & - & $0.009 *(0.005)$ & - \\
\hline \multicolumn{5}{|l|}{ Go-RT var. } \\
\hline Alc. severity & $-0.167^{* *}(0.073)$ & $-0.145 *(0.075)$ & $-0.096(0.074)$ & $-0.157^{* * *}(0.074)$ \\
\hline Smoking (PkYrs) & $-0.014^{* * *}(0.004)$ & $-0.013^{* * *}(0.004)$ & $-0.011^{* *}(0.004)$ & $-0.014^{* * *}(0.004)$ \\
\hline Education & - & $0.036(0.027)$ & - & - \\
\hline $\mathrm{IQ}$ & - & - & $0.023^{* * *}(0.005)$ & - \\
\hline Depression & - & - & - & $-0.013(0.011)$ \\
\hline \multicolumn{5}{|l|}{ SS-RT } \\
\hline Alc. severity & $-0.123 *(0.075)$ & - & $-0.047(0.082)$ & \\
\hline Smoking (PkYrs) & $-0.011^{* *}(0.004)$ & - & $-0.009^{* *}(0.004)$ & \\
\hline IQ & - & - & $0.017^{* * *}(0.005)$ & \\
\hline
\end{tabular}

For each executive function measure, alcoholism severity and pack-years were included only if they had a significant correlation with that variable. Alc.: alcoholism; IQ: intelligence quotient; PkYrs: pack-years. ${ }^{*} P<0.05$; ${ }^{* *} P<0.01$; ${ }^{* * *} P<0.001$. COWAT: Controlled Oral Word Association Test; PASAT: Paced Auditory Serial Addition Task; STROOP-w: Stroop Word Reading Test; STROOP-c: Stroop Color Naming Test; STROOP-cw: Stroop Color Word Interference Test; WCST-cat: Wisconsin Card Sorting Test, categories achieved; TMT-A: Trail Making Test A; Go-RT: Stopping Task go trials reaction-time; Go-RT var.: Stopping Task go trial reaction-time variability; SS-RT: Stopping Task stopping reaction-time; SE: standard error. 
not significant (coeff. $=-0.269, \mathrm{SE}=0.222 ; t=-1.22$, $P=0.223$ ), while both alcoholism severity (coeff. $=$ $-0.128, \mathrm{SE}=0.046 ; t=-2.79, P<0.006)$ and smoking (pack-years) (coeff. $=-0.007, \mathrm{SE}=0.003 ; t=-2.58$. $P<0.011)$ remained significant.

\section{DISCUSSION}

This study follows-up previous work to attempt to isolate associations of alcoholism and smoking with higherorder cognitive abilities. Several interesting results emerged. To begin, among a community-recruited sample of alcoholic men and controls a broad range of EF measures were correlated significantly with alcoholism severity. This extends the results of previous studies with samples recruited from treatment sites, where it is expected that the average severity of alcoholism is higher than our sample. The present data show lower performance in the precise way one would expect, given neuropsychological risk from alcohol use. These participants, while not impaired clinically, are not performing at the level of a carefully neighborhood-matched non-alcoholic group. Furthermore, our results were not explained by levels of ASPD or ADHD covarying with alcoholism severity and smoking. However, the effects of alcoholism severity on EF in our sample were not independent of IQ. Our results could indicate that the $\mathrm{EF}$ effects are subsumed under more general effects on overall intelligence.

The present results also show that smoking is an important variable for some measures of $\mathrm{EF}$ and has a pattern of effects that are distinct from AUD. In particular, smoking was correlated with measures that emphasize response speed. This adds to our previous work and shows that the effects of smoking are quite robust, because they remained significant even with education or IQ included in the regression models. Although we do not know the exact mechanism through which smoking is related to EF, one possibility is that, over time, smoking leads to poor cognitive proficiency. For example, chronic smoking may have damaging effects on the brain via several routes, including neurotoxic action, oxidative stress or by reducing blood flow. Risks for cardiovascular disease [19] and chronic obstructive pulmonary disease (COPD) [45] are increased by smoking. In turn, both cardiovascular disease [46] and COPD [47] are associated with reduced neurocognitive function. It is also possible, but not likely, that nicotine withdrawal contributed to the relationship between smoking and EF performance that we observed.

Previous research has suggested that smoking may mediate the effects of alcoholism on cognitive proficiency [11]. The current results reinforce the importance of smoking when considering cognitive function in alcoholism $[12,13]$, and suggest the possibility that the effect of chronic smoking is primarily to slow information process- ing speed, whereas the effects of alcoholism are more widespread across cognitive domains. The effects of smoking on response execution are consistent with other studies that have linked smoking to reduced informationprocessing speed or efficiency [14,19,21].

Finally, the effects of smoking and alcoholism severity on behavioral inhibition measured by the Stopping Task are new. The existing literature indicates EF deficits in alcoholics from traditional neuropsychological tests that measure more global aspects of EF. The present study demonstrates that a particular component of $\mathrm{EF}$, the ability to inhibit a response, is also impaired in alcoholics and fits well with the view that drug abuse and addiction involve failures of response inhibition [3,48]. Although in this adult sample we cannot determine whether response-inhibition impairments preceded problem alcohol use or were caused by problem alcohol use in our sample, it is none the less a critical finding with regard to EF in alcoholism. With its relation to impulsive, stimulusdriven behavior and the inability to resist short-term gratification, poor response-inhibition may underlie or exacerbate problem alcohol use, and similarly has significant implications for treatment outcomes [49].

\section{Declarations of interest}

None.

\section{Acknowledgements}

This study was supported by NIDA grant R01 DA021032 to J.M. Glass and NIAAA grants R337 AA07065 and AA12217 to R.A. Zucker and J.T. Nigg.

\section{References}

1. Knight R. G., Longmore B. E. Clinical Neuropsychology of Alcoholism. Hove: Lawrence Erlbaum Associates Ltd; 1994.

2. Sullivan E. V., Rosenbloom M. J., Pfefferbaum A. Pattern of motor and cognitive deficits in detoxified alcoholic men. Alcohol Clin Exp Res 2000; 24: 611-21.

3. Giancola P. R., Moss H. B. Executive cognitive functioning in alcohol use disorders. Recent Dev Alcohol 1998; 14: 227-51.

4. Desmond J. E., Chen S. H., DeRosa E., Pryor M. R., Pfefferbaum A., Sullivan E. V. Increased frontocerebellar activation in alcoholics during verbal working memory: an fMRI study. Neuroimage 2003; 19: 1510-20.

5. Adams K. M., Gilman S., Koeppe R. A., Kluin K. J., Brunberg J. A., Dede D. et al. Neuropsychological deficits are correlated with frontal hypometabolism in positron emission tomography studies of older alcoholic patients. Alcohol Clin Exp Res 1993; 17: 205-10.

6. Sullivan E. V., Harding A. J., Pentney R., Dlugos C., Martin P. R., Parks M. H. et al. Disruption of frontocerebellar circuitry and function in alcoholism. Alcohol Clin Exp Res 2003; 27: 301-9.

7. Harper C. The neuropathology of alcohol-specific brain damage, or does alcohol damage the brain? J Neuropathol Exp Neurol 1998; 57: 101-10. 
8. Nigg J. T., Wong M. M., Martel M. M., Jester J. M., Puttler L. I., Glass J. M. et al. Poor response inhibition as a predictor of problem drinking and illicit drug use in adolescents at risk for alcoholism and other substance use disorders. J Am Acad Child Adolesc Psychiatry 2006; 45: 468-75.

9. Nigg J. T., Glass J. M., Wong M. M., Poon E., Jester J. M., Fitzgerald H. E. et al. Neuropsychological executive functioning in children at elevated risk for alcoholism: findings in early adolescence. J Abnorm Psychol 2004; 113: 302-14.

10. Adams K. M., Grant I. Influence of premorbid risk factors on neuropsychological performance in alcoholics. J Clin Exp Neuropsychol 1986; 8: 362-70.

11. Glass J. M., Adams K. M., Nigg J. T., Wong M. M., Puttler L. I., Buu A. et al. Smoking is associated with neurocognitive deficits in alcoholism. Drug Alcohol Depend 2006; 82: 11926.

12. Durazzo T. C., Rothlind J. C., Gazdzinski S., Banys P., Meyerhoff D. J. A comparison of neurocognitive function in nonsmoking and chronically smoking short-term abstinent alcoholics. Alcohol 2006; 39: 1-11.

13. Meyerhoff D. J., Tizabi Y., Staley J. K., Durazzo T. C., Glass J. M., Nixon S. J. Smoking comorbidity in alcoholism: neurobiological and neurocognitive consequences. Alcohol Clin Exp Res 2006; 30: 253-64.

14. Ernst M., Heishman S. J., Spurgeon L., London E. D. Smoking history and nicotine effects on cognitive performance. Neuropsychopharmacology 2001; 25: 313-19.

15. Rezvani A. H., Levin E. D. Cognitive effects of nicotine. Biol Psychiatry 2001; 49: 258-67.

16. Galanis D. J., Joseph C., Masaki K. H., Petrovitch H., Ross G. W., White L. A longitudinal study of drinking and cognitive performance in elderly Japanese American men: the Honolulu-Asia Aging Study. Am J Public Health 2000; 90: 1254-9.

17. Jacobsen L. K., Krystal J. H., Mencl W. E., Westerveld M., Frost S. J., Pugh K. R. Effects of smoking and smoking abstinence on cognition in adolescent tobacco smokers. Biol Psychiatry 2005; 57: 56-66.

18. Hill R. D. Residual effects of cigarette smoking on cognitive performance in normal aging. Psychol Aging 1989; 4: 251-4.

19. Kalmijn S., van Boxtel M. P., Verschuren M. W., Jolles J., Launer L. J. Cigarette smoking and alcohol consumption in relation to cognitive performance in middle age. Am J Epidemiol 2002; 156: 936-44.

20. Richards M., Jarvis M. J., Thompson N., Wadsworth M. E. Cigarette smoking and cognitive decline in midlife: evidence from a prospective birth cohort study. Am J Public Health 2003; 93: 994-8.

21. Cervilla J. A., Prince M., Mann A. Smoking, drinking, and incident cognitive impairment: a cohort community based study included in the Gospel Oak project. J Neurol Neurosurg Psychiatry 2000; 68: 622-6.

22. Pennington B. Dimensions of executive functions in normal and abnormal development. In: Drasnegor N. A., GoldmanRakic P. A., editors. Development of the Prefrontal Cortex: Evolution, Neurobiology, and Behavior. Baltimore, MD: Paul H. Brooks; 1997, p. 265-81.

23. Nigg J. T., Huang-Pollock C. L. An early onset model of the role of executive functions and intelligence in conduct disorder/delinquency. In: Lahey B. B., Moffit T, Caspi A, editors. The Causes of Conduct Disorder and Serious Juvenile Delinquency. New York, NY: The Guildford Press; 2003, p. 227-53.
24. Sattler J. M. Assessment of Children: Cognitive Applications, 4th edn. San Diego: Sattler, J.M.; 2001.

25. Pennington B. F., Ozonoff S. Executive functions and developmental psychopathology. J Child Psychol Psychiatry 1996; 37: 51-87.

26. Ackerman P. L., Beier M. E., Boyle M. O. Working memory and intelligence: the same or different constructs. Psychol Bull 2005; 131: 30-60.

27. Friedman N. P., Miyake A., Corley R. P., Young S. E., Defries J. C., Hewitt J. K. Not all executive functions are related to intelligence. Psychol Sci 2006; 17: 172-9.

28. Fama R., Pfefferbaum A., Sullivan E. V. Perceptual learning in detoxified alcoholic men: contributions from explicit memory, executive function, and age. Alcohol Clin Exp Res 2004; 28: 1657-65.

29. Logan G. D., Cowan W. B., Davis K. A. On the ability to inhibit responses in simple and choice reaction time tasks: a model and a method. J Exp Psychol Hum Percept Perform 1984; 10: 276-91.

30. Osman A., Kornblum S., Meyer D. E. The point of no return in choice reaction time: controlled and ballistic stages of response preparation. J Exp Psychol Hum Percept Perform 1986; 12: 243-58.

31. Nigg J.T. The ADHD response-inhibition deficit as measured by the stop task: replication with DSM-IV combined type, extension, and qualification. J Abnorm Child Psychol 1999; 27: 393-402.

32. Fillmore M. T., Rush C. R. Impaired inhibitory control of behavior in chronic cocaine users. Drug Alcohol Depend 2002; 66: 265-73.

33. Logan G. D., Schachar R. J., Tannock R. Impulsivity and inhibitory control. Psychol Sci 1997; 8: 60-4.

34. Vogel-Sprott M., Easdon C., Fillmore M., Finn P., Justus A. Alcohol and behavioral control: cognitive and neural mechanisms. Alcohol Clin Exp Res 2001; 25: 117-21.

35. Zucker R. A., Fitzgerald H. E., Refior S. K., Puttler L. I., Pallas D. M., Ellis D. A. The clinical and social ecology of childhood for children of alcoholics: description of a study and implications for a differentiated social policy. In: Fitzgerald H. E., Lester B. M., Zuckerman B. S., editors. Children of Addiction: Research, Health and Policy Issues. New York: RoutledgeFalmer; 2000, p. 109-41.

36. Feighner J. P., Robins E., Guze S. B., Woodruff R. A., Jr, Winokur, G., Munoz R. et al. Diagnostic criteria for use in psychiatric research. Arch Gen Psychiatry 1972; 26: 57-63.

37. American Psychiatric Association. Diagnostic and Statistical Manual of Mental Disorders, 4th edn. Washington, DC: American Psychiatric Association; 2000.

38. Lezak M. D., Howieson D. B., Loring D. W. Neuropsychological Assessment, 4th edn. New York, NY: Oxford University Press; 2004.

39. Band G. P., van der Molen M. W., Logan G. D. Horse-race model simulations of the stop-signal procedure. Acta Psychol (Amst) 2003; 112: 105-42.

40. Hamilton M. A rating scale for depression. J Neurol Neurosurg Psychiatry 1960; 23: 56-62.

41. Robins L. N., Helzer J. E., Croughan J., Ratcliff K. S. National Institute of Mental Health Diagnostic Interview Schedule. Its history, characteristics, and validity. Arch Gen Psychiatry 1981; 38: 381-9.

42. Zucker R. A., Ellis D. E., Fitzgerald H. E., Bingham C. R., Sanford K. P. Other evidence for at least two alcoholisms, II. Life course variation in antisociality and heterogeneity of alcoholic outcome. Dev Psychopathol 1996; 8: 831-48. 
43. Reynolds C. R., Wilson V. L., Clark R. L. A four-test short form of the WAIS-R for clinical screening. Clin Neuropsychol 1983; 5: 111-16.

44. Rubin D. B. Multiple Imputation for Nonresponse in Surveys. New York, NY: Wiley; 1987.

45. Sutherland E. R., Martin R. J. Airway inflammation in chronic obstructive pulmonary disease: comparisons with asthma. J Allergy Clin Immunol 2003; 112: 819-27.

46. Geroldi C., Ferrucci L., Bandinelli S., Cavazzini C., Zanetti O., Guralnik J. M. Mild cognitive deterioration with subcortical features: prevalence, clinical characteristics, and association with cardiovascular risk factors in communitydwelling older persons (the InCHIANTI study). J Am Geriatr Soc 2003; 51: 1064-71.
47. Grant I., Prigatano G. P., Heaton R. K., McSweeny A. J., Wright E. C., Adams K. Progressive neuropsychological impairment and hypoxemia: relationship in chronic obstructive pulmonary disease. Arch Gen Psychiatry 1987; 44: 999-1006.

48. Martin C. S., Fillmore M. T., Chung T., Easdon C. M., Miczek K. A. Multidisciplinary perspectives on impaired control over substance use. Alcohol Clin Exp Res 2006; 30: 26571.

49. Bowden S. C., Crews F. T., Bates M. E., Fals-Stewart W., Ambrose M. L. Neurotoxicity and neurocognitive impairments with alcohol and drug-use disorders: potential roles in addiction and recovery. Alcohol Clin Exp Res 2001; 25: 317-21. 